PROCEEDINGS OF THE

AMERICAN MATHEMATICAL SOCIETY

Volume 125, Number 12, December 1997, Pages 3575-3580

S 0002-9939(97)04053-7

\title{
RIGID SETS AND NONEXPANSIVE MAPPINGS
}

\author{
GIOVANNI DILENA, BASILIO MESSANO, AND DELFINA ROUX \\ (Communicated by Palle E. T. Jorgensen)
}

\begin{abstract}
We introduce a new class of normed spaces (not necessarily finite dimensional), which contains the finite dimensional normed spaces with polyhedral norm.

We study the properties of rigid sets of the spaces of this class and we apply the results to limit sets of the sequences of iterates of nonexpansive maps.
\end{abstract}

\section{INTRODUCTION}

Let us consider a compact subset $C$ of a normed space $X$ and a nonexpansive map $f: C \rightarrow C$. Let us denote by $\omega(x)$ the set of limit points of sequence $\left\{f^{n}(x)\right\}$, $x \in C$.

In 1987 Akcoglu and Krengel [1], by using some properties of rigid sets, proved that: if $X=\left(\mathbb{R}^{n},\|\|_{1}\right), n \geq 1$, then $\omega(x)$ is finite, $\forall x \in C$. By a different procedure, Weller [13], extended the above result to any finite dimensional normed space with polyhedral norm. Successively, other authors [5]-[12] also gave an estimate of cardinality of $\omega(x)$ in all these spaces.

In this paper we introduce a suitable class of normed spaces which are not necessarily finite dimensional. We study rigid sets in these spaces and prove that basic properties of rigid sets in $\left(\mathbb{R}^{n},\|\cdot\|_{1}\right)$ still hold in the spaces of the above class. This allows us to obtain some results concerning $\omega(x)$. In particular, Theorem 4.1 gives a new simpler proof of Weller's result, quoted before.

\section{THE $S$-SPACES}

We say that a real normed space $(X,\|\|)$ is an $S$-space if the following condition holds:

(P) For each $a \in X-\{0\}$ there exists $\delta=\delta(a)>0$ such that if $x, y \in X$ and

(i) $\|x\|<\delta,\|y\|<\delta$,

(ii) $\|a-x\| \leq\|a\|,\|a-y\| \leq\|a\|$,

(iii) $\|a-(x+y)\|=\|a\|$,

Received by the editors April 14, 1995 and, in revised form, October 23, 1995, January 22, 1996, and July 3, 1996.

1991 Mathematics Subject Classification. Primary 47H09, 47H07, 47H10.

Key words and phrases. $S$-space, polyhedral norm, rigid sets, nonexpansive mappings, limit sets.

This work was performed under the auspices of M.U.R.S.T.

(C)1997 American Mathematical Society 
then

$$
\|a-x\|=\|a\|, \quad\|a-y\|=\|a\| .
$$

Proposition 2.1. If there exists a sequence $\left\{\varphi_{n}\right\}$ of continuous linear functionals on $(X,\|\|)$ weakly convergent to zero and

$$
\|x\|=\sup _{n}\left|\varphi_{n}(x)\right| \quad \forall x \in X,
$$

then $(X,\|\|)$ is an $S$-space.

Proof. Let $a$ be a point of $X-\{0\}$. Let us set

$$
\delta=\delta(a)=\frac{1}{4} \min \left\{\|a\|, \min \left\{\|a\|-\left|\varphi_{n}(a)\right|:\left|\varphi_{n}(a)\right|<\|a\|\right\}\right\},
$$

and let us assume that $x$ and $y$ belong to $X$ and satisfy (i), (ii), (iii).

If $\left|\varphi_{n}(a)\right|<\|a\|$ we have

$$
\begin{aligned}
\left|\varphi_{n}(a)-\varphi_{n}(x+y)\right| & \leq\left|\varphi_{n}(a)\right|+2 \delta=\left|\varphi_{n}(a)\right|+2 \cdot \frac{1}{4}\left(\|a\|-\left|\varphi_{n}(a)\right|\right) \\
& =\frac{1}{2}\|a\|+\frac{1}{2}\left|\varphi_{n}(a)\right|<\|a\| .
\end{aligned}
$$

Then, by (iii), there exists $m$ such that

$$
\left|\varphi_{m}(a)\right|=\|a\| \quad \text { and }\left|\varphi_{m}(a)-\varphi_{m}(x+y)\right|=\|a\| .
$$

Thus

$$
\varphi_{m}(x)=-\varphi_{m}(y)
$$

and, from (ii), we obtain

$$
\varphi_{m}(x)=\varphi_{m}(y)=0
$$

so (2.1) holds.

Remarks. 1. Hypothesis " $\left\{\varphi_{n}\right\}$ weakly convergent to zero" cannot be weakened. Indeed, let us consider the space $c$ of the convergent sequences of real numbers with sup-norm. Let $a=\left\{\left(1-\frac{1}{n}\right)\right\}_{n \in N}, \delta>0, n_{0}$ an integer greater than $(2 \delta)^{-1}$, $x=y=\left\{x_{n}\right\}_{n \in N}$ where

$$
x_{n}= \begin{cases}\delta\left(1-\frac{1}{n}\right), & n \neq n_{0}, \\ -\frac{1}{2 n_{0}}, & n=n_{0} .\end{cases}
$$

It is easy to see that conditions (i), (ii), (iii) hold, but:

$$
\|a-x\|<1=\|a\| \text {. }
$$

2. The space $c_{0} \subset c$ of the sequences convergent to zero is an $S$-space.

3. A real finite dimensional normed space endowed with a polyhedral norm is an $S$-space.

Proposition 2.2. Let $(X,\|\|)$ be an $S$-space. If $a, x \in X, a \neq 0,\|x\|<\delta(a)$ and $\|a-x\|=\|a\|$, then

$$
\|a-\lambda x\|=\|a\| \quad \forall \lambda \in[0,1] .
$$


Indeed:

$$
\begin{aligned}
& \|a-\lambda x\|=\|\lambda(a-x)+(1-\lambda) a\| \leq\|a\|, \\
& \|a-(1-\lambda) x\|=\|(1-\lambda)(a-x)+\lambda a\| \leq\|a\|, \\
& \|a-[\lambda x+(1-\lambda) x]\|=\|a-x\|=\|a\| .
\end{aligned}
$$

Moreover $\|\lambda x\| \leq\|x\|<\delta(a)$ and $\|(1-\lambda) x\| \leq\|x\|<\delta(a)$; then, from (P), (2.2) holds.

Corollary 2.1. An S-space is not necessarily strictly convex. 1.

Indeed, if $\|a\|=\|b\|=1$ and $\|a-b\|<\delta(a),(2.2)$ gives $\left\|a-\frac{1}{2}(a-b)\right\|=\left\|\frac{a+b}{2}\right\|=$

Remark. In an $S$-space the function $a \rightarrow \delta(a)$ can be discontinuous.

Indeed, in $\left(\mathbb{R}^{2},\|\|_{\infty}\right)$ if $a=(1-\varepsilon, 1), 0<\varepsilon<1$, we have $\delta(a) \leq \varepsilon \rightarrow 0$ if $\varepsilon \rightarrow 0$.

Theorem 2.1. If $(X,\|\|)$ is a finite dimensional real space, then the following statements are equivalent:

(1) For each $a \in X$ there exists $\delta(a)>0$ such that:

$$
\|a\|=\|b\| \text { and }\|b-a\|<\delta(a) \Rightarrow\|\lambda b+(1-\lambda) a\|=\|a\|, \forall \lambda \in] 0,1[.
$$

(2) \|| $\|$ is a polyhedral norm.

(3) $(X,\|\|)$ is an $S$-space.

In order to prove the above theorem we need:

Lemma 2.1. Let $K$ be a nonempty compact convex subset of Euclidean space $\mathbb{R}^{n}$, $n \geq 2$, and let $\Gamma$ be its boundary. If, for each $a \in \Gamma$, there exists $\delta(a)>0$ such that

$$
b \in \Gamma \text { and }\|b-a\|<\delta(a) \Rightarrow \lambda b+(1-\lambda) a \in \Gamma, \forall \lambda \in] 0,1[,
$$

then $K$ is a polytope (of dimension $\leq n$ ).

Indeed, if $n=2$ and $K$ is not a singleton, being $\Gamma$ compact, there exists a finite number $m \geq 1$ of points of $\Gamma$, namely $a_{1}, a_{2}, \ldots, a_{m}$, such that the open balls

$$
S_{i}=\left\{x \in \mathbb{R}^{n}:\left\|x-a_{i}\right\|<\delta\left(a_{i}\right)\right\}, \quad i=1, \ldots, m,
$$

are a finite covering of $\Gamma$. So, by $(2.3), \Gamma \cap S_{i}$ is constituted by at most two segments. Then $\Gamma$ is either a segment or the boundary of a convex polygon (endowed with at most $m$ edges).

If $n>2$, we go on by induction.

Let $a_{1}, a_{2}, \ldots, a_{m}, S_{1}, S_{2}, \ldots, S_{m}$ be as above.

By (2.3), for every $i=1, \ldots, m, \Gamma \cap S_{i}=\gamma_{i} \cap S_{i}$, where $\gamma_{i}$ is the boundary of a (convex) cone $\Gamma_{i}$ obtained by projecting from $a_{i}$ the intersection of $K$ with a suitable hyperplane of $\mathbb{R}^{n}$.

Every nonempty intersection $K^{\prime}$ of $K$ with a hyperplane of $\mathbb{R}^{n}$ lies in $\mathbb{R}^{n-1}$ and satisfies the hypotheses of the lemma; so $K^{\prime}$ is a polytope of dimension $\leq n-1$.

Then $\Gamma_{i}$ is a polyhedral cone (which in some case can degenerate) and $K$ is a polytope of dimension $\leq n$ (with at most $m$ vertices). q.e.d.

Remark. In the case $n>2$ Lemma 2.1 can be proved also by using a result of Klee ([4], Theorem 4.7). 
Proof of Theorem 2.1. (1) $\Rightarrow(2)$ The unit ball $U$ of $X$ is isomorphic to a subset $K$ of an euclidean space $\mathbb{R}^{n}$ which satisfies hypotheses of Lemma 2.1. Then \|\| is a polyhedral norm.

$(2) \Rightarrow(3)$ By Proposition 2.1.

(3) $\Rightarrow(1)$ By Proposition 2.2.

Corollary 2.2. An $S$-space is not smooth.

Indeed, by Theorem 2.1, the intersection of its unit ball with a two-dimensional linear subspace of $X$ is not smooth. Then $X$ is not smooth ([2], (2), p. 112).

\section{Rigid SETS IN AN $S$-SPACE}

Let us recall that a compact set in a metric space $(M, d)$ is called "rigid" if it is the closure of a sequence $\left\{x_{n}\right\}$ in $M$ such that

$$
d\left(x_{n+k}, x_{n}\right)=d\left(x_{k+1}, x_{1}\right), \quad \forall n \geq 1, \forall k \geq 1 .
$$

Rigid sets were studied in the framework of metric spaces by Akcoglu and Krengel [1]; in particular they proved that: rigid sets in $\left(\mathbb{R}^{n},\|\|_{1}\right)$ are finite.

In this section, we extend this result to rigid sets in any finite dimensional $S$ space.

Let us start by proving:

Lemma 3.1. Let $A$ be a rigid set in an $S$-space, $d=\operatorname{diam} A$ and $x, y \in A$ such that

$$
\|x-y\|=d .
$$

Then there exists $\varepsilon=\varepsilon(x-y)$ such that

$$
z \in A \text { and }\|y-z\|<\varepsilon \Rightarrow\|x-z\|=d .
$$

Proof. If $d=0,(3.1)$ is trivial. If $d>0$, there is an isometry $T: A \rightarrow A$ such that $\left\{T^{n}(y)\right\}$ is dense in $A$ (see [1], Lemma 3.2); so, it suffices to prove that (3.1) holds when $z=T^{r}(y)$, for some $r>0$.

Let us set $y-x=a$ and $\varepsilon=\delta(a)$, where $\delta(a)$ is given by $(\mathrm{P})$.

If $\left\|T^{r}(y)-y\right\|<\varepsilon$, from Lemma 3.3 of [1], we also have $\left\|T^{r}(x)-x\right\|<\varepsilon$. Moreover,

$$
\begin{aligned}
& \left\|a-\left(T^{r}(x)-x\right)\right\|=\left\|y-x-T^{r}(x)+x\right\|=\left\|y-T^{r}(x)\right\| \leq\|a\|, \\
& \left\|a-\left(y-T^{r}(y)\right)\right\|=\left\|y-x-y+T^{r}(y)\right\|=\left\|T^{r}(y)-x\right\| \leq\|a\|, \\
& \left\|a-\left[\left(T^{r}(x)-x\right)+\left(y-T^{r}(y)\right)\right]\right\|=\left\|y-x-T^{r}(x)+x-y+T^{r}(y)\right\| \\
& \quad=\left\|T^{r}(y)-T^{r}(x)\right\|=\|y-x\|=\|a\| .
\end{aligned}
$$

Hence, from $(\mathrm{P})$, we have

$$
\left\|T^{r}(y)-x\right\|=\|a\|=d .
$$

Thus the assertion has been proved.

Remark. Lemma 3.1 generalizes Lemma 3.8 of [1] to any $S$-space (both finite and infinite dimensional).

Corollary 3.1. With the hypotheses of Lemma 3.1 the set

$$
\{z \in A:\|z-x\|=d\}
$$

is open on $A$. 
Theorem 3.1. A rigid set in a finite dimensional $S$-space is finite.

Proof. Let $A_{0}$ be a rigid set. Since $A_{0}$ is compact, in order to prove this theorem, it suffices to show that $A_{0}$ is totally disconnected.

Let $a$ be a point of $A_{0}$. By Lemma 3.4 of [1] there exists $a_{0} \in A_{0}$ such that $\left\|a_{0}-a\right\|=\operatorname{diam} A_{0}$.

Let us set

$$
B_{1}=\left\{x \in A_{0}:\left\|x-a_{0}\right\|=\operatorname{diam} A_{0}\right\} .
$$

$B_{1}$ is a closed set, $B_{1}$ is open on $A_{0}$ by Corollary 3.1 and $a \in B_{1}$.

So, by Corollary 3.7 of [1], there exists a rigid set $A_{1}$ open on $A_{0}$, such that $a \in A_{1} \subset B_{1}$.

Then, iterating the above procedure, we obtain a sequence $\left\{a_{n}\right\}$ of points of $A_{0}$ and a sequence $\left\{A_{n}\right\}$ of rigid sets, open on $A_{0}$, such that for each $n \geq 0$

$$
\begin{gathered}
a_{n} \in A_{n}, \quad\left\|a_{n}-a\right\|=\operatorname{diam} A_{n}, \\
a \in A_{n+1} \subset A_{n}, \quad\left\|x-a_{n}\right\|=\operatorname{diam} A_{n} \quad \forall x \in A_{n+1} .
\end{gathered}
$$

Hence the sequence $\left\{x_{n}\right\}$, where $x_{n}=a-a_{n}$, is such that

$$
\left\|x_{m}-x_{n}\right\|=\left\|x_{n}\right\| \quad \forall n \geq 0 \forall m>n .
$$

Then, by Theorem 2 of [3], there exists $h$ such that $\left\|x_{h}\right\|=0$; consequently $A_{h}=\{a\}$. So, since $A_{h}$ is open on $A_{0}, a$ is an isolated point of $A_{0}$.

Thus the assertion has been proved.

Remark. Theorem 3.1 is not true any longer if the $S$-space is infinite dimensional. It is possible to give different counterexamples; we report a simple one given by the referee.

Given the $S$-space $c_{0}$, as an infinite rigid set of $c_{0}$ we can consider the $\omega$-limit set of the sequence $\left\{f^{n}\left(\left\{\frac{1}{k}\right\}\right)\right\},\left\{\frac{1}{k}\right\} \in c_{0}$ and $f: c_{0} \rightarrow c_{0}$ permutes the first two, the next three,..., the next $p_{1}, \ldots$ components, where $p_{i}$ is the $i$-th prime number.

\section{Omega limit sets of nonexpansive mappings in an $S$-SPACE}

As a consequence of Theorem 3.1 we have

Theorem 4.1. Let $C$ be a compact subset of a finite dimensional $S$-space and $f: C \rightarrow C$ be a nonexpansive map. Then, for each $x_{0} \in C, \omega\left(x_{0}\right)$ is finite.

Indeed, $\forall x_{0} \in C, \omega\left(x_{0}\right)$ is a rigid set because the restriction of $f$ to $\omega\left(x_{0}\right)$ is an isometry from $\omega\left(x_{0}\right)$ onto $\omega\left(x_{0}\right)$ such that, $\forall x \in \omega\left(x_{0}\right)$, the sequence $\left\{f^{n}(x)\right\}$ is dense in $\omega\left(x_{0}\right)$ (see [7], p. 523). So, according to Lemma 3.2 of [1], $\omega\left(x_{0}\right)$ is a rigid set.

Corollary 4.1. Let $C$ be a subset of an $S$-space and $f: C \rightarrow C$ be a nonexpansive map. If $x_{0} \in C$ has bounded orbit and $\omega\left(x_{0}\right)$ lies in a finite dimensional linear subspace of $X$, then $\omega\left(x_{0}\right)$ is finite.

Indeed, $\omega\left(x_{0}\right)$ is a closed bounded subset of a finite dimensional $S$-space and $f\left(\omega\left(x_{0}\right)\right)=\omega\left(x_{0}\right)$. Then $\omega\left(x_{0}\right)$ is compact and $\omega(y)=\omega\left(x_{0}\right) \forall y \in \omega\left(x_{0}\right)$. Hence, we can apply Theorem 4.1 to any point $y$ of the compact $\omega\left(x_{0}\right)$ and we obtain the result.

Corollary 4.1 is a local version of Theorem 4.1 which holds also in infinite dimensional $S$-spaces. In general, in these spaces we have only the following result: 
Theorem 4.2. Let $C$ be a compact subset of an $S$-space and $f: C \rightarrow C$ be a nonexpansive map. Then, for each $x_{0} \in C, \omega\left(x_{0}\right)$ is either a singleton or not connected.

Proof. Let $x_{0}$ be a point of $C$ such that $\omega\left(x_{0}\right)$ is not a singleton. Since $\omega\left(x_{0}\right)$ is a rigid set, $\forall a \in \omega\left(x_{0}\right)$, the closed set

$$
H=\left\{x \in \omega\left(x_{0}\right):\|a-x\|=\operatorname{diam} \omega\left(x_{0}\right)\right\}
$$

is open on $\omega\left(x_{0}\right)$ by Corollary 3.1 .

Then the set $K=\omega\left(x_{0}\right)-H$ is closed and $K$ is nonempty because $a \in K$. Then $\omega\left(x_{0}\right)$ is not connected.

\section{REFERENCES}

[1] M. A. Akcoglu and U. Krengel, Nonlinear models of diffusion on a finite space, Prob. Th. Related Fields 76 (1987), 411-420. MR 89b:60167

[2] M. M. Day, Normed linear spaces, Ergebnisse der Mathematik und Ihrer Grenzgebiete, Band. 21, Springer-Verlag, 1973. MR 49:9588

[3] G. DiLena, B. Messano, and D. Roux, Una caratterizzazione degli spazi normati a dimensione infinita, Atti Accad. Sci. Torino Cl. Sci. Fis. Mat. Natur. 128, Fasc. 5-6 (1994). MR 96h:46016

[4] V. Klee, Some characterizations of convex polyhedra, Acta Math. 102 (1959), 79-107. MR 21:4390

[5] U. Krause and R. D. Nussbaum, A limit set trichotomy for self-mappings of normal cones in Banach spaces, Nonlinear Analysis, Theory, Methods \& Applications, Vol. 20, n. 7 (1993), 855-870. MR 94i:47088

[6] R. N. Lions and R. D. Nussbaum, On transitive and commutative finite groups of isometries, Fixed Point Theory and Applications, World Scientific Publ. Co. (1992), 189-229. MR 94a:54096

[7] R. D. Nussbaum, Omega limit sets of nonexpansive maps: finiteness and cardinality estimates, Differential and Integral Equations 3 (1990), 523-540. MR 91f:47079

[8] R. D. Nussbaum, Estimates of the periods of periodic points for nonexpansive operators, Israel J. Math. 76 (1991), 345-380. MR 94a:47094

[9] R. D. Nussbaum, Convergence of iterates of a nonlinear operator arising in statistical mechanics, Nonlinearity 4 (1991), 1223-1240. MR 92j:47103

[10] R. D. Nussbaum, Lattice Isomorphisms and Iterates of Nonexpansive Maps, Nonlinear Analysis, Theory, Methods \& Applications, vol. 22 (1994). MR 95j:47066

[11] R. D. Nussbaum, Finsler structures for the part metric and Hilbert's projective metric and applications to ordinary differential equations, Differential and Integral Equations, vol. 7, n. 6, November 1994, 1649-1707. MR 95b:58010

[12] R. Sine, A nonlinear Perron-Frobenius theorem, Proc. Am. Math. Soc., vol. 109, n. 2, June 1990, 331-336. MR 90i:47058

[13] D. Weller, Hilbert's metric, part metric and self-mappings of a cone, $\mathrm{Ph}$. D. Dissertation, University of Bremen, West Germany, December 1987.

Dipartimento di Matematica dell’Università, Via G. Fortunato, Campus UniversiTARIO - 70125 BARI, ITALY

Dipartimento di Matematica e Applicazioni "R. Caccioppoli" dell'Università, Via Claudio 21 - 80125 Napoli, Italy

Dipartimento di Matematica "F. Enriques" Dell'Università, Via Saldini 50 - 20133 Milano, Italy

E-mail address: roux@vmimat.mat.unimi.it 